\title{
Morbilidad psiquiátrica en consumidores de cocaína y heroína reclutados en la comunidad
}

\section{Psychiatric morbidity among cocaine and heroin users in the community}

\author{
Silvia TortajadA*; Ma Jesús Herrero*; Antònia Domingo- \\ SalvanY", ${ }^{* *}$; GeMma Molist ${ }^{*},{ }^{* * *}$; Gregorio BarRIO ${ }^{*}, * * ;$ \\ Luís de LA FUENTE ${ }^{*},{ }^{* \star *} ;$ M $^{a}$ TeRESA BrUGaL ${ }^{*}{ }^{* \star *} ;$ GRUPO dE \\ INVESTIGACIÓN ITINERE
}

${ }^{*}$ CIBER en Epidemiología y Salud Pública (CIBERESP). ${ }^{\star}$ Drug Abuse Epidemiology Research Group. IMIM-Hospital del Mar. ${ }^{* \star *}$ Centro Nacional de Epidemiología. Instituto de Salud Carlos III. ${ }^{\star \star \star \star}$ Agència de Salut Pública de Barcelona (ASPB).

\section{Resumen}

El consumo de drogas es un problema de salud pública. Entre los consumidores la presencia concomitante de trastornos mentales y abuso de sustancias son comunes. Este artículo examina los trastornos psiquiátricos que presentan jóvenes consumidores de cocaína y heroína, medidos con la World Mental Health Composite International Diagnostic Interview (WMH-CIDI).

Se realizó una encuesta transversal de entrada a una cohorte de 1.266 jóvenes (18-30 años), consumidores regulares de cocaina y no heroína (705) y de 561 consumidores de heroína reclutados fuera de los servicios de salud en Barcelona, Madrid y Sevilla. Para evaluar los trastornos mentales se utilizó el WMH-CIDI, para medir el grado de dependencia la Severity of Dependence Scale (SDS), y para evaluar el apoyo social se utilizó el Cuestionario de Apoyo Social Funcional Duke-UNC.

Se diagnosticó un trastorno mental al 43\% de la muestra. Los diagnósticos más frecuentes fueron la depresión $(37,5 \%)$ y la fobia especifica $(6,8 \%)$. Durante los últimos 12 meses, la prevalencia de trastorno mental en el grupo de heroína (26.4\%) fue mayor que en el de cocaina (21.7\%). Las variables relacionadas con la morbilidad psiquiátrica en la cohorte de cocaína fueron: el mayor número de días de consumo, las condiciones de vida inestables y el bajo apoyo social. En la cohorte de heroína, el ganar dinero a través de actividades ilegales, mientras que el consumo moderado de alcohol actuó como factor protector para la patologia mental. En ambas cohortes, la morbilidad se asoció a haber recibido tratamiento psiquiátrico/psicológico durante los últimos 12 meses.

Este estudio muestra una prevalencia relativamente alta de morbilidad psiquiátrica en consumidores de cocaina y heroina reclutados en entornos no clínicos. Para el futuro, son necesarios estudios que examinen las diferencias entre los patrones de consumo de cocaína y heroína y su asociación con las enfermedades mentales.

Palabras clave: cocaina, heroína, CIDI, morbilidad psiquiátrica, población juvenil.

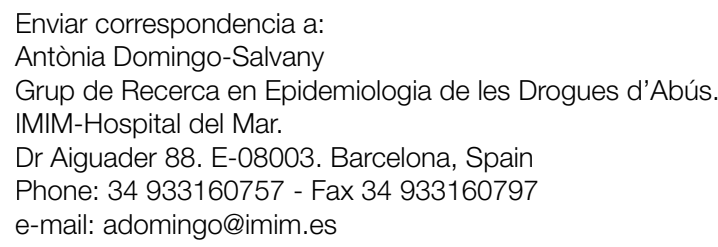

\section{Abstract}

Drug abuse is a serious public health problem. Moreover, co-occurring mental health and substance abuse disorders are common among drug users. This paper examines psychiatric disorders of young cocaine and heroin users using the World Mental Health Composite International Diagnostic Interview (WMH-CIDI).

A cohort of 1266 young (18-30 years) current regular cocaine (705) and heroin (561) users were recruited outside the health services in Barcelona, Madrid and Seville, Spain. The WMH-CIDI was used to evaluate mental disorders; the Severity of Dependence Scale (SDS) measured the degree of dependence; and the Duke-UNC Functional Social Support Questionnaire (FSSO) assessed social support, in a crosssectional study design.

About $43 \%$ was diagnosed with a lifetime mental disorder. The most common diagnoses were depression (37.5\%) and specific phobia (6.8\%). During the last 12 months, prevalence rates were also slightly higher in heroin group (26.4\%) than in cocaine cohort (21.7\%). Every day cocaine consumption, having unstable living conditions and low social support were variables highly associated with psychiatric morbidity in cocaine cohort. In heroin cohort, earning money through illegal activities was associated with psychiatric morbidity, while the moderate use of alcohol acted as a protective factor for mental pathology. Morbidity was associated to having received psychiatric/psychological treatment during the last 12 months in both cohorts.

This study has shown a relatively high prevalence of psychiatric morbidity in cocaine and heroin users recruited in non-clinical settings. Future studies examining differences between cocaine and heroin patterns of consumption associated with mental diseases are necessary.

Key words: cocaine, heroin, CIDI, psychiatric morbidity, young population. 
T e term 'comorbidity' was introduced in medicine to denote those cases in which a 'distinct additional clinical entity' occurred during the clinical course of a patient having an index disease (Feinstein, 1970). In psychiatry, this term indicates not only those cases in which a patient receives both a psychiatric and a general medical diagnosis (e.g. major depression and diabetes), but also those cases in which a patient receives two or more psychiatric diagnoses (e.g. major depression and panic disorder). This co-occurrence of two or more psychiatric diagnoses ('psychiatric comorbidity' or 'dual diagnosis') has been reported to be very frequent. Prevalence rates range from $21 \%$ to $54 \%$ depending on the population studied, the time frame assessed, and methodology and tools used to obtain diagnoses (Alonso y Lepine, 2007; Andrews, Slade, \& Issakidis, 2002; Casares-Lopez et al., 2011; Krueger, Chentsova-Dutton, Markon, Goldberg, \& Ormel, 2003; Roncero et al., 2011; Szerman et al., 2011; Torrens, Gilchrist, \& Domingo-Salvany, 2011) .

Within the field of 'psychiatric comorbidity' that encompasses a wide range of potential concomitant psychiatric diagnoses, the term 'dual diagnosis' is defined generically as the co-existence of substance use disorder (SUD) and other psychiatric disorder (non-SUD). Evidence from community and clinical samples has shown that the coexistence of SUD and non-SUD is common (prevalence rates ranging from 3\% to 67\%) (Grant et al., 2004; Kessler, Chiu, Demler, Merikangas, \& Walters, 2005; Pavarin, 2006; Rush et al., 2008).

It is now well established that concomitant substance dependence is associated with a more severe course of independent mental disorders, and that these long term independent syndromes produce greater difficulty in treating the associated substance use disorders (Rounsaville, 2004; van Os et al., 2002). Hence the importance of a careful initial evaluation with an eye toward commonly correlated conditions and a treatment strategy designed to ameliorate all problems, not just the predominant diagnosis.

Dual pathology is usually studied in populations that have already received a SUD diagnosis (abuse or dependence), or in samples recruited from clinical settings. In the present study, the population was recruited outside the health-care services and not all participants suffering from a mental disorder had an additional diagnosis of SUD, despite having a regular substance use. This is the reason why we use the term 'psychiatric morbidity'.

The aim of the present study was to determine the psychiatric morbidity in regular cocaine and heroin users recruited outside the clinical setting, using the Composite International Diagnostic Interview (CIDI).

\section{Method}

\section{Subjects}

Young adult cocaine and heroin users were obtained from the ITINERE cohort. The ITINERE project is a cohort study in young cocaine and heroin users in three Spanish cities:
Barcelona, Madrid and Seville (de la Fuente et al., 2005; Pulido et al., 2009). All participants were recruited outside the health-care services by targeted sampling and chain referral methods (Hartnoll et al., 1997; Watters \& Biernacki, 1989). At entry, inclusion criteria for both cohorts, cocaine and heroin, were age (18-30 years); to be resident in the metropolitan area of Barcelona, Madrid or Seville at least 6 month of the last year; being a fluent Spanish speaker; and current (having used heroin or cocaine at least once within the 90 days prior to the interview) and regular use (in heroin subcohort, having taken heroin at least 60 days over the 12 months prior to the interview; and in cocaine subcohort, having used cocaine at least 52 days over the year prior to the interview, in which heroin was taken no more than 12 days). All participants provided written informed consent. The study design was approved by the Institutional Bioethics Committee.

Participants were recruited from June 2001 to July 2006. All data were collected trough a Computer-Assisted Personal Interview (CAPI) (Newman et al., 2002; Turner et al., 1998) programmed using the Questionnaire Development System (ODS) software (NOVA Research Company, 2002). Participation incentive was $18 €$ per interview completed.

The CIDI questions were included in both cohorts from February of 2003 to November of 2006. Cocaine cohort included CIDI in the Baseline Questionnaire, and heroin cohort included CIDI in the first follow-up evaluation.

\section{Measures}

Main socio-demographic characteristics were assessed, emphasizing living conditions (i.e., type of housing and occupational status). Regarding substance use, severity of substance dependence (with the the Spanish version of the Severity of Dependence Scale [SDS]) (Gonzalez-Saiz \& Salvador-Carulla, 1998; Gossop, Best, Marsden, \& Strang, 1997), patterns and circumstances of cocaine and heroin use over the 12 months prior to the interview, and the degree of alcohol use were assessed. Alcohol consumption was rated as moderate (that is, $<40 \mathrm{~g} /$ day for men and $<20 \mathrm{~g} /$ day for women), at risk (40-80 g/day for men and 20-40 g/day for women), or heavy ( $\geq 80 \mathrm{~g} /$ day for men and $\geq 40 \mathrm{~g} /$ day for women)(Rehm, 1998).

Psychiatric morbidity was assessed using the World Mental Health (WMH) Composite International Diagnostic Interview (CIDI) (Alonso et al., 2002; Kessler \& Ustun, 2004), a comprehensive, fully structured, lay-administered interview developed by the World Health Organization that generates diagnoses according to ICD-10 and DSM-IV diagnostic criteria (Robins et al., 1988). Although this version of the interview was designed to assess the Axis I major mental disorders, dimensions of health-related quality of life, and the burden imposed by illness on the family, in the present study the sections of WMH-CIDI administered were the most frequently found in this kind of substance users: depression, panic disorder, specific phobia, social phobia, and agoraphobia. The CIDI was translated to Spanish taking into account European Study of Epidemiology of Mental Disorders (ESEMeD) project recommendations (Alonso \& Lepine, 2007) and it has proved 
to be both reliable and valid (Haro et al., 2006). In the present study, we used lifetime and current (last 12 months) diagnostics according to DSM-IV criteria.

The Duke-UNC Functional Social Support Questionnaire (FSSO) (Broadhead, Gehlbach, de Gruy, \& Kaplan, 1988) was administered to measure cocaine and heroin users' perception of personal social support. The FSSO is a self-report questionnaire composed of 8 items that assess two factors: Confidant Support and Affective Support. This version with 8 items has been validated in Spanish general population (Bellon Saameno, Delgado, Luna del Castillo, \& Lardelli, 1996; Lahuerta, Borrell, Rodriguez-Sanz, Perez, \& Nebot, 2004).

\section{Data analysis}

Prior to statistical analysis, all the variables were examined through various programs for accuracy of data entry and missing values, and fit between their distributions and assumptions of multivariate analysis. The statistical significance of differences in the qualitative variables was assessed with Chi-square and Fisher exact tests and, in the quantitative variables, with analysis of variance (ANOVA). Significant values were set at $p<0.05$ with two-tailed tests.

A logistic regression model was fitted to assess the influence of other variables on psychiatric morbidity. The Hosmer-Lemeshow statistic was used to assess the goodnessof-fit. Variables were selected from those showing a $p<0.10$ in the univariate analysis, avoiding the simultaneous introduction of those highly correlated (i.e.: SDS and patterns of use).

Cocaine and heroin cohorts were analyzed separately because distribution of different variables differed among subjects from the two cohorts and they showed different patterns of relationship to psychiatric morbidity. This allowed comparisons between cohorts. SPSS (version 15) was used for all analyses.

\section{Results}

\section{Socio-demographic characteristics}

Of the 1266 subjects, 69\% were men. The mean age of cocaine and heroin users was 23 years (standard deviation [SD] 3.3), and 26.0 years (SD 3.2), respectively. In both cohorts women were younger than men $(p<0.019$ and $p<0.0001$, respectively). Nearly $77 \%$ had completed more than primary education, more frequently subjects in cocaine cohort (87.2\% vs. $63.1 \%$ in heroin cohort) ( $p<0.001$ ). The $41.5 \%$ was unemployed, mainly in heroin cohort (59.9\% vs $26.9 \%$ ) ( $p<$ 0.001 ). Nearly $20.5 \%$ had ever been in jail or prison, being this percentage higher in heroin users $(41.5 \%$ vs $3.7 \%)(p<0.001)$. The type of residence was also different depending on the cohorts: participants from heroin cohort presented unstable living conditions (homeless, institutions, squatters, and so on) more frequently than cocaine users $(29.6 \%$ vs. $11.2 \%) \quad(p<$ 0.001) (Table 1).

Regarding social support (assessed via FSSO), cocaine cohort had higher scores (better) on all scales (affective support, confidant support and overall score) than subjects from heroin cohort $(p<0.005)$ (Table 1$)$.

\section{Patterns of use}

In heroin cohort, only $3.1 \%$ had not used cocaine over the last 12 months, whereas $84.5 \%$ of users from cocaine cohort had not used heroin during the previous year ( $p<$ 0.001 ) (Table 1). Snorting was the main route of cocaine use in cocaine cohort $(92 \%)$ ( $p<0.001)$; in heroin cohort, nearly $61 \%$ smoked heroin without statistically significant differences as compared to the rest of routes of administration. Of note, in cocaine cohort nearly $21 \%$ of subjects had smoked cocaine over the last 12 months whereas in heroin cohort this figure was $65.2 \%$ ( $p<0.001)$. Moreover, in heroin cohort nearly $46 \%$ had injected any substance during the last 12 months, and only $4 \%$ in cocaine cohort $(p<0.001)$.

About 59\% of the subjects from cocaine group used this substance one or two days a week or less, and $2.8 \%$ every day. Nearly $41 \%$ of the subjects from heroin cohort used heroin one or two days a week and $16.2 \%$ every day (Table 1 ).

Regarding alcohol consumption, higher proportion of nondrinkers was found in heroin cohort (25\% vs. 4.7\%). Overall, cocaine cohort showed a higher consumption at risk level (34.3\% vs. $12.12 \%$ in heroin cohort) ( $<<0.001)$. As assessed by SDS, a greater severity of heroin (heroin cohort: mean 4.9, SD 4.4; cocaine cohort: mean 0.2, SD 1.2) and cocaine dependence (heroin cohort: mean 4.7, SD 4.2; cocaine cohort: mean 4.3, SD 3.0) was found in heroin cohort. Differences in severity of heroin and cocaine dependence between both cohorts were statistically significant $(p<0.001)$ (Table 1).

Overall, most subjects (77.9\%) had not received any kind of treatment during the last 12 months, 3.3\% had received psychiatric or psychological treatment, $15.7 \%$ treatment for drug use, and only $3.1 \%$ had received both. More subjects from heroin cohort (32\%) than those from cocaine group $(2.8 \%)$ had received any substance-related treatment during the previous year $(p<0.001)$ (Table 1$)$.

\section{Psychiatric morbidity}

Overall, $43 \%$ of the sample was diagnosed with a lifetime mental disorder other than a substance use disorder, with the highest prevalence rates in heroin group (47\% vs. $40.4 \%$ in cocaine cohort, $p<0.005)$. During the last 12 months, prevalence rates were lower than lifetime ones, being slightly higher again in heroin group (26.4\% vs $21.7 \%$ in cocaine cohort) $(p<0.005)$. Lifetime major depression was the most frequent diagnosis in both cohorts (37.5\%), followed by specific phobia (6.8\%) and panic disorder (6.3\%) (Table 2). 
Table 1. Sociodemographic characteristics and drug use patterns, according to cocaine and heroin cohorts and current psychiatric morbidity.

\begin{tabular}{|c|c|c|c|c|c|c|c|c|c|c|c|c|c|c|c|c|}
\hline & & & & \multicolumn{6}{|c|}{ Cocaine } & \multicolumn{7}{|c|}{ Heroin } \\
\hline & \multicolumn{3}{|c|}{ Total $^{a}$} & \multicolumn{4}{|c|}{ Morbidity } & \multirow{2}{*}{\multicolumn{2}{|c|}{ Total $^{b}$}} & \multicolumn{4}{|c|}{ Morbidity } & \multirow{2}{*}{\multicolumn{3}{|c|}{ Total $^{b}$}} \\
\hline & & & & \multicolumn{2}{|c|}{ Yes } & \multicolumn{2}{|c|}{ No } & & & \multicolumn{2}{|l|}{ Yes } & \multicolumn{2}{|c|}{ No } & & & \\
\hline & \multicolumn{3}{|c|}{$n=1266$} & $n=153$ & (21.7\%) & $n=552$ & (78.3\%) & \multicolumn{2}{|c|}{$n=705(55.7 \%)$} & $n=148$ & $(26.4 \%)$ & $n=41$ & $3(73.6 \%)$ & $n=561$ & (44.3\%) & \\
\hline Gender & & & $* *$ & & & & & & $* * *$ & & & & & & & $* * *$ \\
\hline Men & 873 & $(69 \%)$ & & 71 & $(15.2 \%)$ & 397 & $(84.8 \%)$ & 468 & $(66.4 \%)$ & 85 & $(21.0 \%)$ & 320 & $(79.0 \%)$ & 405 & $(72.2 \%)$ & \\
\hline Women & 393 & $(31 \%)$ & & 82 & $(34.6 \%)$ & 155 & $(65.4 \%)$ & 237 & $(33.6 \%)$ & 63 & $(40.4 \%)$ & 93 & $(59.6 \%)$ & 156 & $(27.8 \%)$ & \\
\hline Age (years) & & & $* * *$ & & & & & & & & & & & & & ** \\
\hline$<=25$ & 743 & $(58.7 \%)$ & & 114 & $(21.4 \%)$ & 418 & $(78.6 \%)$ & 532 & $(75.5 \%)$ & 66 & $(31.3 \%)$ & 145 & $(68.7 \%)$ & 211 & $(37.6 \%)$ & \\
\hline$>25$ & 523 & $(41.3 \%)$ & & 39 & $(22.5 \%)$ & 134 & $(77.5 \%)$ & 173 & $(24.5 \%)$ & 82 & $(23.4 \%)$ & 268 & $(76.6 \%)$ & 350 & $(62.4 \%)$ & \\
\hline Educational level & & & $* * *$ & & & & & & & & & & & & & \\
\hline$\leq$ Primary level & 297 & $(23.5 \%)$ & & 21 & $(23.3 \%)$ & 69 & $(76.7 \%)$ & 90 & $(12.8 \%)$ & 49 & $(23.7 \%)$ & 158 & $(76.3 \%)$ & 207 & $(36.9 \%)$ & \\
\hline$\geq$ Secondary level & 969 & $(76.5 \%)$ & & 132 & $(21.5 \%)$ & 483 & (78.5\%) & 615 & $(87.2 \%)$ & 99 & $(28.0 \%)$ & 255 & $(72.0 \%)$ & 354 & (63.1\%) & \\
\hline Occupational status ${ }^{1}$ & & & $* * *$ & & & & & & ** & & & & & & & ** \\
\hline Employed & 504 & $(39.8 \%)$ & & 70 & $(20.8 \%)$ & 266 & $(79.2 \%)$ & 336 & $(47.7 \%)$ & 35 & $(20.8 \%)$ & 133 & $(79.2 \%)$ & 168 & $(29.9 \%)$ & \\
\hline Unemployed & 526 & $(41.5 \%)$ & & 54 & $(28.4 \%)$ & 136 & $(71.6 \%)$ & 190 & $(27 \%)$ & 89 & $(26.5 \%)$ & 247 & $(73.5 \%)$ & 336 & $(59.9 \%)$ & \\
\hline Other ${ }^{2}$ & 236 & $(18.6 \%)$ & & 29 & $(16.2 \%)$ & 150 & $(83.8 \%)$ & 179 & $(25.4 \%)$ & 24 & $(42.1 \%)$ & 33 & $(57.9 \%)$ & 57 & $(10.2 \%)$ & \\
\hline Type of housing ${ }^{1}$ & & & $* *$ & & & & & & *** & & & & & & & \\
\hline $\begin{array}{l}\text { Individual or familial housing } \\
\text { Homeless / institutions / }\end{array}$ & 1020 & $(80.6 \%)$ & & 122 & $(19.5 \%)$ & 503 & $(80.5 \%)$ & 625 & $(88.7 \%)$ & 95 & $(24.1 \%)$ & 300 & $(75.9 \%)$ & 395 & $(70.4 \%)$ & \\
\hline no fixed abode / squatters & 246 & $(19.4 \%)$ & & 31 & $(38.8 \%)$ & 49 & $(61.3 \%)$ & 80 & $(11.2 \%)$ & 53 & $(31.9 \%)$ & 113 & $(68.1 \%)$ & 166 & $(29.6 \%)$ & \\
\hline $\begin{array}{l}\text { Confidant support (FSSO) }{ }^{+1} \\
\text { (mean, [sd]) }\end{array}$ & 71.3 & {$[25.0]$} & ** & 73.7 & {$[21.4]$} & 78.6 & {$[20.1]$} & 77.6 & {$[20.5]$} & 61.5 & {$[28.1]$} & 64.1 & {$[27.7]$} & 63.4 & [27.8] & * \\
\hline $\begin{array}{l}\text { Affective support (FSSO) }{ }^{1} \\
\text { (mean, [sd]) }\end{array}$ & 77.7 & [24.4] & $* *$ & 75.3 & [25.2] & 82.2 & [18.9] & 80.7 & [20.6] & 72 & [27.2] & 74.5 & [28.2] & 73.8 & [28.0] & $* * *$ \\
\hline $\begin{array}{l}\text { Overall score FSSQ } \\
\text { (mean, }[\mathrm{sd}])\end{array}$ & 73.7 & {$[22.7]$} & $* *$ & 74.3 & {$[20.7]$} & 80 & {$[17.7]$} & 78.8 & {$[18.5]$} & 65.4 & {$[25.3]$} & 68.0 & {$[25.9]$} & 67.3 & {$[25.7]$} & $* * *$ \\
\hline Smoked cocaine use ${ }^{1}$ & & & $* * *$ & & & & & & ** & & & & & & & \\
\hline No & 753 & $(59.5 \%)$ & & 107 & $(19.2 \%)$ & 451 & $(80.8 \%)$ & 558 & $(79.1 \%)$ & 48 & $(24.6 \%)$ & 147 & $(75.4 \%)$ & 195 & $(34.8 \%)$ & \\
\hline Yes & 513 & $(40.5 \%)$ & & 46 & $(31.3 \%)$ & 101 & $(68.7 \%)$ & 147 & $(20.9 \%)$ & 100 & $(27.3 \%)$ & 266 & $(72.7 \%)$ & 366 & $(65.2 \%)$ & \\
\hline Injected ${ }^{1}$ & & & $* * *$ & & & & & & $* * *$ & & & & & & & \\
\hline No & 982 & $(77.6 \%)$ & & 138 & $(20.4 \%)$ & 539 & $(79.0 \%)$ & 677 & $(96 \%)$ & 77 & $(25.2 \%)$ & 228 & $(74.8 \%)$ & 305 & $(54.4 \%)$ & \\
\hline Yes & 284 & $(22.4 \%)$ & & 15 & $(53.6 \%)$ & 13 & $(46.4 \%)$ & 28 & $(4 \%)$ & 71 & $(27.7 \%)$ & 185 & $(72.3 \%)$ & 256 & $(45.6 \%)$ & \\
\hline Frequency of cocaine use ${ }^{1}$ & & & $* * *$ & & & & & & & & & & & & & \\
\hline Never & 17 & $(1.3 \%)$ & & & & & & & $* * *$ & 3 & $(17.6 \%)$ & 14 & $(82.4 \%)$ & 17 & $(3.1 \%)$ & \\
\hline Every day & 169 & $(13.4 \%)$ & & 12 & $(60.0 \%)$ & 8 & $(40.0 \%)$ & 20 & $(2.8 \%)$ & 31 & $(20.8 \%)$ & 118 & $(79.2 \%)$ & 149 & $(26.8 \%)$ & \\
\hline Weekly & 441 & $(35 \%)$ & & 65 & $(24.0 \%)$ & 206 & $(76.0 \%)$ & 271 & $(38.5 \%)$ & 48 & $(28.2 \%)$ & 122 & $(71.8 \%)$ & 170 & $(30.6 \%)$ & \\
\hline 1-2 times a week or less & 633 & $(50.2 \%)$ & & 75 & $(18.2 \%)$ & 338 & $(81.8 \%)$ & 413 & $(58.7 \%)$ & 66 & $(30.0 \%)$ & 154 & $(70.0 \%)$ & 220 & $(39.6 \%)$ & \\
\hline Frequency of heroin use ${ }^{1}$ & & & & & & & & & $* * *$ & & & & & & & \\
\hline Never & & & & 113 & $(19.2 \%)$ & 477 & $(80.8 \%)$ & 590 & $(84.5 \%)$ & & & & & & & \\
\hline 1-2 times a week or less & & & & 38 & $(35.2 \%)$ & 70 & $(64.8 \%)$ & 108 & $(15.5 \%)$ & & & & & & & \\
\hline Frequency of heroin use ${ }^{1}$ & & & & & & & & & & & & & & & & \\
\hline Never & & & & & & & & & & 30 & $(25.0 \%)$ & 90 & $(75.0 \%)$ & 120 & $(21.4 \%)$ & \\
\hline Every day & & & & & & & & & & 17 & $(18.7 \%)$ & 74 & $(81.3 \%)$ & 91 & $(16.2 \%)$ & \\
\hline Weekly & & & & & & & & & & 31 & $(25.6 \%)$ & 90 & $(74.4 \%)$ & 121 & $(21.6 \%)$ & \\
\hline 1-2 times a week or less & & & & & & & & & & 70 & $(30.6 \%)$ & 159 & $(69.4 \%)$ & 229 & $(40.8 \%)$ & \\
\hline Amount of alcohol (ml/day) ${ }^{1}$ & & & $* * *$ & & & & & & & & & & & & & \\
\hline None & 173 & $(13.7 \%)$ & & 11 & $(33.3 \%)$ & 22 & $(66.7 \%)$ & 33 & $(4.7 \%)$ & 43 & $(30.7 \%)$ & 97 & $(69.3 \%)$ & 140 & $(25 \%)$ & \\
\hline Moderate use & & & & & & & & & & & & & & & & \\
\hline $\begin{array}{l}\text { (<40 male / } 20 \text { female gr/day) } \\
\text { At-risk use }\end{array}$ & 415 & $(32.8 \%)$ & & 35 & $(19.8 \%)$ & 142 & $(80.2 \%)$ & 177 & $(25.1 \%)$ & 50 & $(21.0 \%)$ & 188 & $(79.0 \%)$ & 238 & $(42.4 \%)$ & \\
\hline ( $40-80$ male/20-40 female gr/day) & 310 & $(24.5 \%)$ & & 43 & $(17.8 \%)$ & 199 & $(82.2 \%)$ & 242 & $(34.3 \%)$ & 17 & $(25.0 \%)$ & 51 & $(75.0 \%)$ & 68 & $(12.1 \%)$ & \\
\hline $\begin{array}{l}\text { Heavy use } \\
\text { (>80 male/ } 20 \text { female } \mathrm{gr} / \mathrm{day})\end{array}$ & 368 & $(29.1 \%)$ & & 64 & $(25.3 \%)$ & 189 & $(74.7 \%)$ & 253 & $(35.9 \%)$ & 38 & $(33.0 \%)$ & 77 & $(67.0 \%)$ & 115 & $(20.5 \%)$ & \\
\hline $\mathrm{SDS}^{+}$- Heroin ${ }^{1}($ mean $[\mathrm{sd}])$ & 2.3 & [3.9] & *** & 0.4 & {$[1.5]$} & 0.2 & {$[1.1]$} & 0.2 & {$[1.2] *$} & 4.8 & {$[4.1]$} & 4.9 & {$[4.5]$} & 4.9 & {$[4.4]$} & \\
\hline SDS - Cocaine ${ }^{1}($ mean $[s d])$ & 4.5 & {$[3.6]$} & *** & 5.5 & {$[3.9]$} & 3.9 & {$[2.8]$} & 4.3 & {$[3.0] * * *$} & 5.1 & {$[4.2]$} & 4.6 & {$[4.3]$} & 4.7 & {$[4.2]$} & \\
\hline Treatment ${ }^{1}$ & & & *** & & & & & & $* * *$ & & & & & & & \\
\hline None & 986 & $(77.9 \%)$ & & 123 & $(19.0 \%)$ & 523 & $(81.0 \%)$ & 646 & $(91.6 \%)$ & 85 & $(25.0 \%)$ & 255 & $(75.0 \%)$ & 340 & $(60.6 \%)$ & \\
\hline Psychological/psychiatric treatment & 42 & $(3.3 \%)$ & & 16 & $(55.2 \%)$ & 13 & $(44.8 \%)$ & 29 & $(4.1 \%)$ & 7 & $(53.8 \%)$ & 6 & $(46.2 \%)$ & 13 & $(2.3 \%)$ & \\
\hline Drug treatment & 199 & $(15.7 \%)$ & & 6 & $(30.0 \%)$ & 14 & $(70.0 \%)$ & 20 & $(2.8 \%)$ & 46 & $(25.7 \%)$ & 133 & $(74.3 \%)$ & 179 & $(31.9 \%)$ & \\
\hline Both & 39 & $(3.1 \%)$ & & 8 & $(80.0 \%)$ & 2 & $(20.0 \%)$ & 10 & $(1.4 \%)$ & 10 & $(34.5 \%)$ & 19 & $(65.5 \%)$ & 29 & $(5.2 \%)$ & \\
\hline
\end{tabular}

Note.

during the last 12 months; ${ }^{2}$, Others, include students, pensioners and subjects with illegal activities.

${ }^{+}$FSSO: Duke-UNC Functional Social Support Questionnaire. SDS: Severity Dependence Scale.

a asterisks under this column reflect statistical significance between cohorts (cocaine and heroin); ${ }^{b}$ asterisks under these columns reflect statistical significance between presence and absence of comorbidity.

${ }^{*} P<0.01 ;{ }^{* *} P<0.005 ;{ }^{* * *} P<0.001$ 
Table 2. Axis I disorders in cocaine and heroin cohorts by gender.

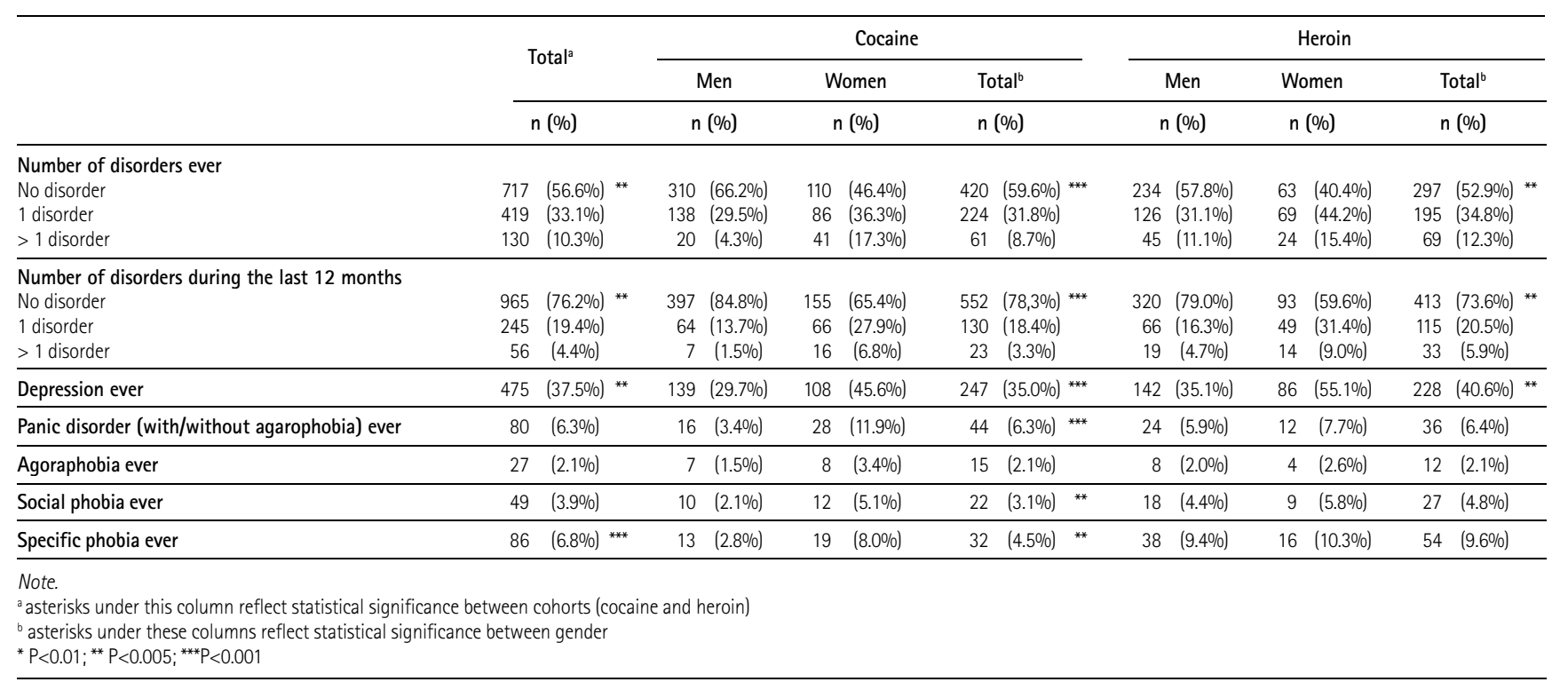

Among those subjects that presented at least one disorder, the mean number of mental disorders diagnosed in cocaine users was 1.2 (SD 0.55) and in heroin cohort was 1.32 (SD $0.72)(p=0.121)$.

Mental disorders were more frequent in women than in men. Significant differences by gender were found in cocaine cohort for all disorders, except for agoraphobia, and only depression was significantly more prevalent among women in heroin cohort $(p<0.005)$ (Table 2).

Unemployed subjects presented similar prevalence rates of psychiatric morbidity in both cohorts (26.5\% in heroin vs. $28.4 \%$ in cocaine cohort). However, in heroin cohort, users engaged in other activities (mainly, illegal activities) showed a higher prevalence of mental pathology (42.1\%) whereas it was $16.2 \%$ for those in other activities (mainly, students) in cocaine cohort. In both cohorts, people without a stable living situation or those living on the street, was the most affected by mental pathology, with the highest figures observed in cocaine cohort (38.8\% vs. $31.9 \%$ in heroin cohort) $(p<0.001)$ (Table 1).

Regarding route of administration, subjects from cocaine cohort that smoked (20.8\%) and/or injected the drug (4\%) presented psychiatric morbidity more frequently than those who did not in this cohort $(p<0.005$ and $p<0.001$, respectively). Also in cocaine cohort, subjects that used the drug every day or those who used higher amounts of substance (data not shown) had a higher risk of suffering mental pathology, with a dose-response effect in both situations (both, $p<0.001$ ). These patterns were not observed in heroin cohort (Table 1).

As far as alcohol consumption is concerned, subjects from cocaine cohort that did not use alcohol at all, showed the highest rate of mental pathology (33.3\%), and then, subjects with high risk level of alcohol consumption (25.3\%). Something similar although in a different order occurred in heroin cohort: the highest rate of mental pathology was found among subjects with high risk level of alcohol consumption (33\%), followed by those who did not used alcohol at all (30.7\%) (Table 1).

In both cohorts, among those subjects with psychiatric morbidity, the proportion of subjects that had received psychological/psychiatric treatment, plus or not drug related treatment, during the last 12 months was higher than that of subjects that had never received treatment, though only statistically significant among cocaine users (Table 1).

In the multivariate analysis, apart from gender and having received psychiatric/psychological treatment that were significant for current psychiatric morbidity presence in both cohorts, in cocaine cohort, a variable highly associated with psychiatric morbidity was everyday cocaine consumption (adjusted odd ratio [AOR], 6.5; 95\% Cl, 2.0-21.4). Other variables associated with mental pathology were living in squatter communities, in institutions or being people with no fixed abode (AOR, 2.4; 95\% Cl, 1.3-4.1); and social support, so that people with less support had a higher risk for mental pathology (AOR, 0.98; 95\% Cl, 0.97-0.99) (Table 3).

In heroin cohort, psychiatric morbidity slightly decreased with age (AOR, 0.93; 95\% Cl, 0.88-0.99), and increased with earning money through illegal activities (including criminal acts, drug dealing, prostitution, etc) ( $\mathrm{AOR}, 2.2 ; 95 \% \mathrm{Cl}, 1.1-4.4)$ and, compared to non drinkers, light drinkers (that is, subjects that reported a prudent alcohol intake) had a decreased risk of mental pathology (AOR, 0.6; $95 \mathrm{Cl}, 0.3-0.9)$ (Table 3).

\section{Discussion}

In this sample of young regular cocaine or heroin users recruited outside the clinical setting, about 43\% (95\% Cl: 40.6-46\%) were diagnosed with an Axis I mental disorder 
Table 3. Logistic regression analysis for current psychiatric morbidity by cocaine and heroin cohorts.

\begin{tabular}{|c|c|c|c|c|c|}
\hline & Morbidity $\mathrm{N}=153$ & $\mathrm{OR}^{+}$ & $95 \% \mathrm{Cl}^{+}$ & $\mathrm{AOR}^{+a}$ & $95 \% \mathrm{Cl}$ \\
\hline \multicolumn{6}{|l|}{ Cocaine } \\
\hline \multicolumn{6}{|l|}{ Gender } \\
\hline Men & $71(46.4 \%)$ & 1 & & 1 & \\
\hline Women & $82(53.6 \%)$ & 3 & $(2.0-4.3)$ & 3.6 & $(2.4-5.4)$ \\
\hline Age & $23.3[3.2]$ & 1.04 & $(0.98-1.09)$ & 1.03 & $(0.97-1.09)$ \\
\hline \multicolumn{6}{|l|}{ Type of housing ${ }^{1}$} \\
\hline \multicolumn{6}{|l|}{ Individual or familia } \\
\hline \multicolumn{6}{|l|}{$\begin{array}{l}\text { Homeless/institutions/ } \\
\text { no fixed abode/ }\end{array}$} \\
\hline squatters & 31 (20.3\%) & 2.6 & $(1.6-4.3)$ & 2.4 & $(1.3-4.1)$ \\
\hline \multicolumn{6}{|l|}{ Injected ${ }^{1}$} \\
\hline No & $138(90.2 \%)$ & 1 & & 1 & \\
\hline Yes & $15(9.8 \%)$ & 4.5 & $(2.1-9.7)$ & 2.2 & $(0.9-5.6)$ \\
\hline \multicolumn{6}{|c|}{ Frequency of cocaine use ${ }^{1}$} \\
\hline Every day & $12(7.9 \%)$ & 6.8 & $(2.7-17.1)$ & 6.5 & $(2.0-21.4)$ \\
\hline Weekly & $65(42.8 \%)$ & 1.4 & $(1.0-2.1)$ & 1.2 & $(0.8-1.8)$ \\
\hline \multicolumn{6}{|l|}{ 1-2 times a week or } \\
\hline less & 75 (49.3\%) & 1 & & 1 & \\
\hline Overall score $\mathrm{FSSQ}^{+1}$ & $74.3[20.7]$ & 0.98 & $(0.97-0.99)$ & 0.98 & $(0.97-0.99)$ \\
\hline \multicolumn{6}{|l|}{ Treatment $^{1}$} \\
\hline None & $123(80.4 \%)$ & 1 & & 1 & \\
\hline \multicolumn{6}{|l|}{ Psychological/ } \\
\hline psychiatric treatment & $16(10.5 \%)$ & 5.3 & $(2.5-11.2)$ & 4.3 & $(1.9-9.8)$ \\
\hline Drug treatment & $6(3.9 \%)$ & 1.8 & $(0.7-4.8)$ & 0.6 & $(0.2-2.3)$ \\
\hline Both & $8(5.2 \%)$ & 17 & $(3.6-81.1)$ & 17.2 & $(3.3-91.5)$ \\
\hline \multicolumn{2}{|l|}{ Heroin } & $N=148$ & & & \\
\hline \multicolumn{6}{|l|}{ Gender } \\
\hline Men & $85(57.4 \%)$ & 1 & & 1 & \\
\hline Women & $63(42.6 \%)$ & 2.6 & $(1.7-3.8)$ & 2.5 & $(1.6-3.8)$ \\
\hline Age & $25.4[3.5]$ & 0.93 & $(0.88-0.98)$ & 0.93 & $(0.88-0.99)$ \\
\hline \multicolumn{6}{|l|}{ Occupational status ${ }^{1}$} \\
\hline Employed & $35(23.6 \%)$ & 1 & & 1 & \\
\hline Unemployed & 89 (60.1\%) & 1.4 & $(0.9-2.1)$ & 1.1 & $(0.7-1.7)$ \\
\hline Other & $24(16.2 \%)$ & 2.8 & $(1.5-5.3)$ & 2.2 & $(1.1-4.4)$ \\
\hline \multicolumn{6}{|l|}{$\begin{array}{l}\text { Amount of alcohol } \\
\left(\mathrm{ml} / \text { day }^{1}\right.\end{array}$} \\
\hline None & $43(29.1 \%)$ & 1 & & 1 & \\
\hline \multicolumn{6}{|l|}{ Moderate use } \\
\hline 20 female gr/day) & 50 (33.8\%) & 0.6 & $(0.4-1.0)$ & 0.6 & $(0.3-0.9)$ \\
\hline \multicolumn{6}{|c|}{ At-risk use $(40-80$ male } \\
\hline \multirow{2}{*}{\multicolumn{6}{|c|}{ Heavy use (>80 male) }} \\
\hline & & & & & \\
\hline 40 female gr/day) & $38(25.7 \%)$ & 1.1 & $(0.7-1.9)$ & 1 & $(0.6-1.7)$ \\
\hline \multicolumn{6}{|l|}{ Treatment ${ }^{1}$} \\
\hline None & 85 (57.4\%) & 1 & & 1 & \\
\hline \multicolumn{6}{|l|}{ Psychological/ } \\
\hline psychiatric treatment & $7(4.7 \%)$ & 3.5 & $(1.2-10.7)$ & 3.5 & $(1.1-11.2)$ \\
\hline Drug treatment & $46(31.1 \%)$ & 1 & $(0.7-1.6)$ & 1.2 & $(0.7-1.8)$ \\
\hline Both & $10(6.8 \%)$ & 1.6 & $(0.7-3.5)$ & 1.8 & $(0.8-4.1)$ \\
\hline
\end{tabular}

Note.

during the last 12 months

$\mathrm{OR}=$ odds ratio estimate; $\mathrm{Cl}=$ confidence interval $; \mathrm{AOR}=$ Adjusted odds ratio;

FSSO: Duke-UNC Functional Social Support Questionnaire.

Logistic regression

Hosmer-Lemeshow cocaine $c^{2}=6.452, p=0.597$

Hosmer-Lemeshow heroin $c^{2}=5.869, p=0.662$

using mood and anxiety specific sections of the CIDI. Overall, lifetime depression was the most frequent diagnosis (37.5\%), followed by specific phobia (6.8\%) and panic disorder (6.3\%). Mental disorders were more frequent in women than in men. On the one hand, everyday cocaine consumption, having received psychiatric/psychological treatment during the last 12 months, having unstable living conditions and low social support were the variables highly associated with current psychiatric morbidity in cocaine cohort. On the other hand, in heroin cohort, having received only psychiatric/psychological treatment during the last 12 months and earning money through illegal activities were associated with psychiatric morbidity, while being a light drinker seemed to act as a protective factor for mental pathology.
It is worth mentioning that the present work is very different from most of the earlier studies of psychopathology among substance users, because it has focused specifically on cocaine or heroin users who were recruited outside the health-care services. So, one of the strengths of this study is that the findings provide additional perspective on a form of drug use that is entrenched in the street drug scene. A relevant difference from clinical studies is that this sample of young cocaine and heroin consumers included a larger proportion of women (Roncero et al., 2011; Szerman et al., 2012; Torrens et al., 2011). Thus, caution is needed when extrapolating these data to other samples of cocaine or heroin users. Furthermore, because data are cross-sectional, it is not possible to make casual inferences, that is, to know to what extent the psychiatric morbidity has been influenced by drug use and vice versa. Likewise, most parameters in the study were selfreported and recall bias could be expected. However, some previous studies have shown that assuring confidentiality and anonymity of the data (as it was done in the present study) maximizes subjects' response accuracy. In this way, the information obtained from substance users tend to be reliable and valid (Del Boca \& Darkes, 2003).

The relatively high prevalence of mental pathology found in our study (43\%) is consistent with those from previous studies, in which prevalence rates of psychiatric morbidity and comorbidity range from $21 \%$ to $54 \%$ depending on the population studied, the time framework assessed, and methodology and tools used to obtain diagnoses (Alonso \& Lepine, 2007; Andrews et al., 2002; Compton, Thomas, Conway, \& Colliver, 2005; de Girolamo et al., 2005; Grant et al., 2004; Kessler et al., 2005; Krueger et al., 2003; Pavarin, 2006; Rush et al., 2008). Furthermore, as expected, these prevalence rates were considerably higher than those reported in more recent general population-based studies using the CIDI (Alonso et al., 2004; Stein et al., 2008; Williams et al., 2008), except for agoraphobia in Williams' study.

In our context, two studies whose subjects were recruited from our wider sample, have also highlighted the relatively high prevalence of lifetime psychiatric comorbidity (42.4\%, 95\% Cl: $34-51 \%$ in cocaine users; and $67.1 \%, 95 \% \mathrm{Cl}$ : $59.6-$ $74.7 \%$ in heroin users) (Herrero, Domingo-Salvany, Torrens, Brugal, \& and the ITINERE Investigators., 2008; RodriguezLlera et al., 2006). When compared to the present study (43\%), these figures were consistent in the cocaine study, being significantly higher in heroin study (probably because the high prevalence of subjects with personality disorders, unfortunately not included in our study). As compared to specific diagnoses, all of them were higher in our study. So, caution is needed when interpreting these results. These differences probably can be explained by the instrument used to evaluate DSM diagnoses, since in the above mentioned studies the interview (i.e., the Psychiatric Research Interview for Substances and Mental Diseases [PRISM]) was administered by an experienced psychologist applying his clinical judgment to make critical decisions when it was required. It would seem plausible that clinical criteria on the PRISM are more conservative and restrictive than self-reported information from subjects on 
the CIDI. Despite these differences, and consistently with our results, in cocaine study, the most common Axis I disorders were mood disorders (26.6\%) and anxiety disorders (13\%). In heroin study, apart from personality disorders that were the most common, the same pattern was observed (mood disorders, 25\%; and anxiety disorders, 16.8\%).

Taken together, these findings suggest an urgent need for validating the CIDI specifically in substance-abusing population, although this instrument has shown to be a reliable and valid measure in epidemiological studies (Wittchen, 1994).

It is noteworthy that $57 \%$ and $80 \%$ of heroin and cocaine users, respectively, with morbidity in the last 12 months, during that period did not receive any psychiatrist or psychological treatment, neither for drug abuse nor for their possible mental disorder. For cocaine users this figure differed statistically from that of subjects without morbidity (they had contacted treatment even less). It is difficult to compare these results with other studies as, usually, results stem from community or treatment samples. In a similar study with a subsample of cocaine users, lifetime treatment (both for substance use and/or mental disorders) was more common among subjects with lifetime comorbidity (62\% vs $39 \%$ in those without comorbidity) (Herrero et al., 2008), whereas in the study with heroin users subsample, only psychiatric/psychological treatment was more frequent among subjects with lifetime comorbidity (Rodriguez-Llera et al., 2006).

This study adds to the evidence suggesting an association between the prevalence of common mental disorders and gender. Both general population and treatment samples show that rates of psychiatric comorbidity are significantly higher for women than for men (Brady \& Randall, 1999) (Compton, III et al., 2000; Kessler et al., 2005; Rodriguez-Llera et al., 2006) (Alonso \& Lepine, 2007). Specifically, comorbidity rates are higher for mood and anxiety disorders. A recent study on psychiatric comorbidity incidence also showed a higher incidence rate for women(Herrero, Domingo-Salvany, Brugal, \& Torrens, 2011). A systematic review of studies published in the English language since approximately 1990 dealing with comorbid psychiatric and substance use disorders concluded that psychiatric comorbidity affects treatment outcome in distinct ways according to gender (Zilberman, Tavares, \& el Guebaly, 2003). At least for the comorbidity between depression and alcohol use disorders (most of the literature has focused on this substance), the prognosis for women with comorbidity is better than that for their counterparts without comorbidity, while the opposite is true for men. So, further studies are needed to clarify the nature of this relation.

Specifically in heroin cohort, a potential protective benefit of alcohol was observed. It has been suggested that moderate alcohol use compared with non-use was associated with a reduction in mental health admissions (Tait \& Hulse, 2006). These data are consistent with other data from the general population, where moderate drinkers have a lower risk of symptoms of depression and anxiety than either non-users or heavy drinkers (Rodgers et al., 2000). So, and consistently with our findings, there is tentative evidence for more favorable physical, mental and cognitive health in moderate drinkers compared with both problem drinkers and abstainers (the so-called J-shaped curve of alcohol use). These authors have speculated that any association between moderate alcohol use and improved health may be mediated by better general or cardiovascular health, psychological well-being or increased social stability. Non-alcohol use by a subject could be related to a previous physic or psychiatric disorder prompting to abstinence (Kerr, Greenfield, Bond, Ye, \& Rehm, 2011)

On the other hand, in several health-related domains, it is well established that appropriate levels of perceived social support exert a beneficial effect, probably by strengthening internal resources (Alvarez-Ude, Valdes, Estebanez, \& Rebollo, 2004; Cox et al., 2008; Malta, Strathdee, Magnanini, \& Bastos, 2008). Not surprisingly, this is also the case in our sample, with a higher perceived level of social support in cocaine users compared to the heroin group.

Also of major concern was that psychiatric morbidity in our sample of cocaine users seems to be more strongly associated with use-related variables, showing a doseresponse effect, whereas in the heroin group, psychiatric morbidity seems to be more independent from patterns of substance use. In univariate analyses, SDS both for heroin and for cocaine were statistically significant factors related to morbidity among cocaine users though not included in the final logistic regression as SDS-cocaine was correlated with cocaine frequency of use. In the heroin group variables associated with morbidity are more the kind of contextual factors (i.e., type of housing) than substance use related; among them neither frequency nor quantity of cocaine use was associated to psychiatric morbidity. Heroin users, apart from experiencing more mental and legal problems and poorer social and occupational functioning than cocaine group, more frequently used routes of substance administration that pose greater health risks to users (i.e. injecting, smoking).

\section{Conclusions}

In spite of the intensive efforts made until now in politics involving both, health prevention and health resources management, this study reveals some factors that are hardly addressed by current prevention programs and could inform future initiatives. Programs can be targeted at young people who we identified to be at risk, especially when the majority of these substance users do not perceive a need for care and do not seek help from mental health professionals. So, encouraging effective efforts to reduce the progression from use to problematic use (in cocaine group) and directing resources into helping some of the most vulnerable and excluded members of society (in heroin group) would be two approaches that probably need to be intensified.

\section{Acknowledgements}

ITINERE Group includes: M José Bravo; Dani Lacasa; Yolanda Castellanos; José Pulido; David Fernández; Rosario 
Ballesta; Sofía Ruiz; Fermín Fernández; Fernando Sánchez; Fernando Vallejo; Sara Santos; Albert Sanchez-Niubó; Anna Guitart; Montserrat Bartroli and Albert Espelt.

We gratefully acknowledge the contribution of all the participants and of those people and institutions who were involved in recruitment and follow-up-(a) in Madrid: Fundación de Ayuda contra la Drogadicción (FAD), Programas Municipales de Sida e Instituto de las Adicciones, Médicos del Mundo, CAID de Torrejón de Ardoz, Centro Sanitario Sandoval, Subdirección General de Sanidad de Instituciones Penitenciarias, Agencia Antidroga; (b) in Barcelona: ABD, Spott, Ambit, CECAS, Secretaria de Serveis Penitenciaris, Rehabilitació i Justícia Juvenil.

This research was supported by Fundación para la Investigación y Prevención del Sida (Spanish Foundation for AIDS Research and Prevention-FIPSE 36253/01), and grants FIS 01/0908 and G03/005 (Cooperative Investigation Net). The funding sources had no involvement in any phase of the design, data collection or data analysis and interpretation.

\section{Conflict of interest}

Authors declare that they don't have any conflict of interest.

\section{References}

Alonso, J., Angermeyer, M. C., Bernert, S., Bruffaerts, R., Brugha, T. S., Bryson, H.,... Vollebergh. W. A. (2004). Prevalence of mental disorders in Europe: results from the European Study of the Epidemiology of Mental Disorders (ESEMeD) project. Acta Psychiatrica Scandinavica, 109, 21-27.

Alonso, J., Ferrer, M., Romera, B., Vilagut, G., Argermeyer, M., Bernert, S.,... McColgen, Z. (2002). The European study of the epidemiology of mental disorders (ESEMeD/MHEDEA 2000) Project: rationale and methods. International Journal of Methods in Psychiatric Research, 11, 55-67.

Alonso, J. \& Lepine, J. P. (2007). Overview of Key Data From the European Study of the Epidemiology of Mental Disorders (ESEMeD). Journal of Clinical Psychiatry, 68, 3-9.

Alvarez-Ude, F., Valdes, C., Estebanez, C. \& Rebollo, P. (2004). Healthrelated quality of life of family caregivers of dialysis patients. Journal of Nephrology, 17, 841-850.

Andrews, G., Slade, T. \& Issakidis, C. (2002). Deconstructing current comorbidity: data from the Australian National Survey of Mental Health and Well-Being. British Journal of Psychiatry, 181, 306-314.

Bellon Saameno, J. A., Delgado, S. A., Luna del Castillo, J. D. \& Lardelli, C. P. (1996). Validez y fiabilidad del cuestionario de apoyo social funcional Duke-UNC-11. Atencion Primaria, 18, 153-163.

Brady, K. T. \& Randall, C. L. (1999). Gender differences in substance use disorders. Psychiatric Clinics of North America, 22, 241-252.
Broadhead, W. E., Gehlbach, S. H., de Gruy, F. V. \& Kaplan, B. H. (1988). The Duke-UNC Functional Social Support Questionnaire. Measurement of social support in family medicine patients. Medical Care, 26, 709-723.

Casares-Lopez, M. J., Gonzalez-Menendez, A., Bobes-Bascaran, M. T., Secades, R., Martinez-Cordero, A. \& Bobes, J. (2011). Need for the assessment of dual diagnosis in prisons. Adicciones, 23,37-44.

Compton, W. M., III, Cottler, L. B., Ben Abdallah, A., Phelps, D. L., Spitznagel, E. L. \& Horton, J. C. (2000). Substance dependence and other psychiatric disorders among drug dependent subjects: race and gender correlates. The American Journal on Addictions, 9, 113125.

Compton, W. M., Thomas, Y. F., Conway, K. P. \& Colliver, J. D. (2005). Developments in the epidemiology of drug use and drug use disorders. American Journal of Psychiatry, 162, 1494-1502.

Cox, J. E., Buman, M., Valenzuela, J., Joseph, N. P., Mitchell, A. \& Woods, E. R. (2008). Depression, parenting attributes, and social support among adolescent mothers attending a teen tot program. Journal of Pediatric and Adolescent Gynecology, 21, 275-281.

de Girolamo, G., Polidori, G., Morosini, P., Mazzi, F., Serra, G., Scarpino, $V_{.}, . .$. Rossi, A. (2005). [Prevalence of common mental disorders in Italy, risk factors, health status, and utilization of health services: the ESEMeD-WMH project]. Epidemiologia e Psichiatria Sociale, 14, $1-100$.

de la Fuente, L., Brugal, M. T., Ballesta, R., Bravo, M. J., Barrio, G., Domingo-Salvany, A.,... Ambros, M. (2005). Metodología del estudio de cohortes del proyecto ITINERE sobre consumidores de heroína en tres ciudades españolas y caracteristicas básicas de los participantes. Revista Española de Salud Pública, 79, 475-491.

Del Boca, F. K. \& Darkes, J. (2003). The validity of self-reports of alcohol consumption: state of the science and challenges for research. Addiction, 98, 1-12.

Feinstein, A. R. (1970). The pre-therapeutic classification of co-morbidity in chronic disease. Journal of Chronic Diseases, 23, 455-469.

Gonzalez-Saiz, F. \& Salvador-Carulla, L. (1998). Estudio de fiabilidad y validez de la versión española de la escala Severity of Dependence Scale (SDS) [Fiability and validity Study of Spanish version of Severity of Dependence Scale (SDS)]. Adicciones, 10, 223-232.

Gossop, M., Best, D., Marsden, J. \& Strang, J. (1997). Test-retest reliability of the Severity of Dependence Scale. Addiction, 92, 353.

Grant, B. F., Stinson, F. S., Dawson, D. A., Chou, S. P., Dufour, M. C., Compton, W.,... Kaplan, K. (2004). Prevalence and co-occurrence of substance use disorders and independent mood and anxiety disorders: results from the National Epidemiologic Survey on Alcohol and Related Conditions. Archives of General Psychiatry, 61, 807-816.

Haro, J. M., Arbabzadeh-Bouchez, S., Brugha, T. S., de Girolamo, G., Guyer, M. E., Jin, R.,... Kessler, R. C. (2006). Concordance of the Composite International Diagnostic Interview Version 3.0 (CIDI 3.0) with standardized clinical assessments in the WHO World Mental Health surveys. International Journal of Methods in Psychiatric Research, 15, 167-180. 
Hartnoll, R., Griffits, P., Taylor, C., Hendrick, V., Blanken, P., \& Nolimal, D. (1997). Handbook on snowball sampling. Strasbourg: Pompidou Group, Council of Europe.

Herrero, M. J., Domingo-Salvany, A., Brugal, M. T. \& Torrens, M. (2011). Incidence of psychopathology in a cohort of young heroin and or cocaine users. Journal of Substance Abuse and Treatment, 41, $55-63$.

Herrero, M. J., Domingo-Salvany, A., Torrens, M., Brugal, M. T. \& and the ITINERE Investigators. (2008). Psychiatric comorbidity in young cocaine users: induced versus independent disorders. Addiction, 103, 284-293.

Kerr, W. C., Greenfield, T. K., Bond, J., Ye, Y. \& Rehm, J. (2011). Racial and ethnic differences in all-cause mortality risk according to alcohol consumption patterns in the national alcohol surveys. American Journal of Epidemiology, 174, 769-778.

Kessler, R. C., Chiu, W. T., Demler, O., Merikangas, K. R. \& Walters, E. E. (2005). Prevalence, severity, and comorbidity of 12-month DSM-IV disorders in the National Comorbidity Survey Replication. Archives of General Psychiatry, 62, 617-627.

Kessler, R. C. \& Ustun, T. B. (2004). The World Mental Health (WMH) Survey Initiative Version of the World Health Organization (WHO) Composite International Diagnostic Interview (CIDI). International Journal of Methods in Psychiatric Research, 13, 93-121.

Krueger, R. F., Chentsova-Dutton, Y. E., Markon, K. E., Goldberg, D. \& Ormel, J. (2003). A cross-cultural study of the structure of comorbidity among common psychopathological syndromes in the general health care setting. Journal of Abnormal Psychology, 112, 437-447.

Lahuerta, C., Borrell, C., Rodriguez-Sanz, M., Perez, K. \& Nebot, M. (2004). [The influence of the social network on mental health in the elderly]. Gaceta Sanitaria, 18, 83-91.

Malta, M., Strathdee, S. A., Magnanini, M. M. \& Bastos, F. I. (2008). Adherence to antiretroviral therapy for human immunodeficiency virus/acquired immune deficiency syndrome among drug users: a systematic review. Addiction, 103, 1242-1257.

Newman, J. C., Des, J., Turner, C. F., Gribble, J., Cooley, P. \& Paone, D. (2002). The differential effects of face-to-face and computer interview modes. American Journal of Public Health, 92, 294-297.

NOVA Research Company (2002). Questionnaire Development System 2.0 (ODSTM). [Computer software]. Bethesda, NOVA Research Company..

Pavarin, R. M. (2006). Substance use and related problems: a study on the abuse of recreational and not recreational drugs in Northern Italy. Annali dell Istituto Superiore di Sanita, 42, 477-484.

Pulido, J., Teresa, B. M., de la, F. L., Ballesta, R., Barrio, G., Bravo, M. J.,... Fernandez, F. (2009). [Recruitment methodology and characteristics of a cohort of young regular cocaine users in three Spanish cities (the Itinere-cocaine Project).]. Gaceta Sanitaria, 23, 200-207.

Rehm, J. (1998). Measuring quantity, frequency, and volume of drinking. Alcoholism-Clinical and Experimental Research, 22, 4S-14S.

Robins, L. N., Wing, J., Wittchen, H. U., Helzer, J. E., Babor, T. F., Burke, J.,... Regier, D. A. (1988). The Composite International Diagnostic Interview. An epidemiologic Instrument suitable for use in conjunction with different diagnostic systems and in different cultures. Archives of General Psychiatry, 45, 1069-1077.

Rodgers, B., Korten, A. E., Jorm, A. F., Jacomb, P. A., Christensen, H. \& Henderson, A. S. (2000). Non-linear relationships in associations of depression and anxiety with alcohol use. Psychological Medicine, 30, 421-432.

Rodriguez-Llera, M. C., Domingo-Salvany, A., Brugal, M. T., Silva, T. C., Sanchez-Niubo, A. \& Torrens, M. (2006). Psychiatric comorbidity in young heroin users. Drug and Alcohol Dependence, 84, 48-55.

Roncero, C., Daigre, C., Gonzalvo, B., Valero, S., Castells, X., Grau-Lopez, $L, \ldots$ Casas, M. (in press). Risk factors for cocaine-induced psychosis in cocaine-dependent patients. European Psychiatry.

Rounsaville, B. J. (2004). Treatment of cocaine dependence and depression. Biological Psychiatry, 56, 803-809.

Rush, B., Urbanoski, K., Bassani, D., Castel, S., Wild, T. C., Strike, C.,... Somers, J. (2008). Prevalence of co-occurring substance use and other mental disorders in the Canadian population. Canadian Journal of Psychiatry, 53, 800-809.

Stein, D. J., Seedat, S., Herman, A., Moomal, H., Heeringa, S. G., Kessler, R. C. \& Williams D. R. (2008). Lifetime prevalence of psychiatric disorders in South Africa. The British Journal of Psychiatry, 192, 112117.

Szerman, N., Arias, F., Vega, P., Babin, F., Mesias, B., Basurte, I.,... Poyo, F. (2011). Pilot study on the prevalence of dual pathology in community mental health and substance misuse services in Madrid. Adicciones, 23, 249-255.

Szerman, N., Lopez-Castroman, J., Arias, F., Morant, C., Babin, F., Mesias, B.,... Baca-Garcia, E. (2012). Dual diagnosis and suicide risk in a Spanish outpatient sample. Substance Use and Misuse, 47, 383-389.

Tait, R. J. \& Hulse, G. K. (2006). Hospital morbidity and alcohol consumption in less severe psychiatric disorder: 7-year outcomes. The British Journal of Psychiatry, 188, 554-559.

Torrens, M., Gilchrist, G. \& Domingo-Salvany, A. (2011). Psychiatric comorbidity in illicit drug users:substance induced versus independent disorders. Drug and Alcohol Dependence, 113, 147156.

Turner, C. F., Ku, L., Rogers, S. M., Lindberg, L. D., Pleck, J. H. \& Sonenstein, F. L. (1998). Adolescent sexual behavior, drug use, and violence: increased reporting with computer survey technology. Science, 280, 867-873.

van Os, J., Bak, M., Hanssen, M., Bijl, R. V., de Graaf, R. \& Verdoux, H. (2002). Cannabis use and psychosis: a longitudinal populationbased study. American Journal of Epidemiology, 156, 319-327.

Watters, J. K. \& Biernacki, P. (1989). Targeted sampling: options for the study of hidden populations. Social Problems, 36, 416-430.

Williams, D. R., Herman, A., Stein, D. J., Heeringa, S. G., Jackson, P. B., Moomal, H. \& Kessler, R. C. (2008). Twelve-month mental disorders in South Africa: prevalence, service use and demographic correlates in the population-based South African Stress and Health Study. Psychological Medicine, 38, 211-220. 
Wittchen, H. U. (1994). Reliability and validity studies of the WHO-Composite International Diagnostic Interview (CIDI): a critical review. Journal of Psychiatric Research, 28, 57-84.

Zilberman, M., Tavares, H. \& el Guebaly, N. (2003). Gender similarities and differences: the prevalence and course of alcohol- and other substance-related disorders. Journal of Addictive Disorders, 22, $61-74$. 